\title{
Molecular Beam Epitaxy of Two-Dimensional Vanadium-Molybdenum Diselenide Alloys
}

Lei Zhang, ${ }^{\dagger,+}, \Delta$ Tong Yang, ${ }^{\dagger, \Delta}$ Xiaoyue He,${ }^{\dagger}{ }^{\S}$ Wen Zhang, ${ }^{\ddagger, \dagger}$ Giovanni Vinai, ${ }^{*}$ Chi Sin

Tang, ${ }^{\dagger} \%$ Xinmao Yin, ${ }^{\dagger},{ }^{\perp}$ Piero Torelli, ${ }^{\#}$ Yuan Ping Feng, ${ }^{\dagger}$ Ping Kwan Johnny Wong, $, \ldots, * *$ and Andrew T. S. Wee $e^{\dagger, t, *}$

${ }^{\dagger}$ Department of Physics, National University of Singapore, 2 Science Drive 3, Singapore 117542, Singapore

Centre for Advanced 2D Materials (CA2DM) and Graphene Research Centre (GRC), National University of Singapore, 6 Science Drive 2, Singapore 117546, Singapore

${ }^{\S}$ Songshan Lake Materials Laboratory, Dongguan 523808, China

${ }^{\ddagger}$ School of Electronics and Information, Northwestern Polytechnical University, Xi'an 710072, P.R. China

\#Instituto Officina dei Materiali (IOM)-CNR, Laboratorio TASC, Area Science Park, S.S. km 163.5, Trieste I-34149, Italy

${ }^{\%}$ NUS Graduate School for Integrative Sciences and Engineering, National University of Singapore, 117456, Singapore, Singapore

${ }^{\perp}$ Singapore Synchrotron Light Source (SSLS), National University of Singapore, 5 Research Link, Singapore 117603, Singapore

*E-mail: pingkwanj.wong@nwpu.edu.cn; phyweets@nus.edu.sg 


\section{XAS and XMCD measurement geometry}

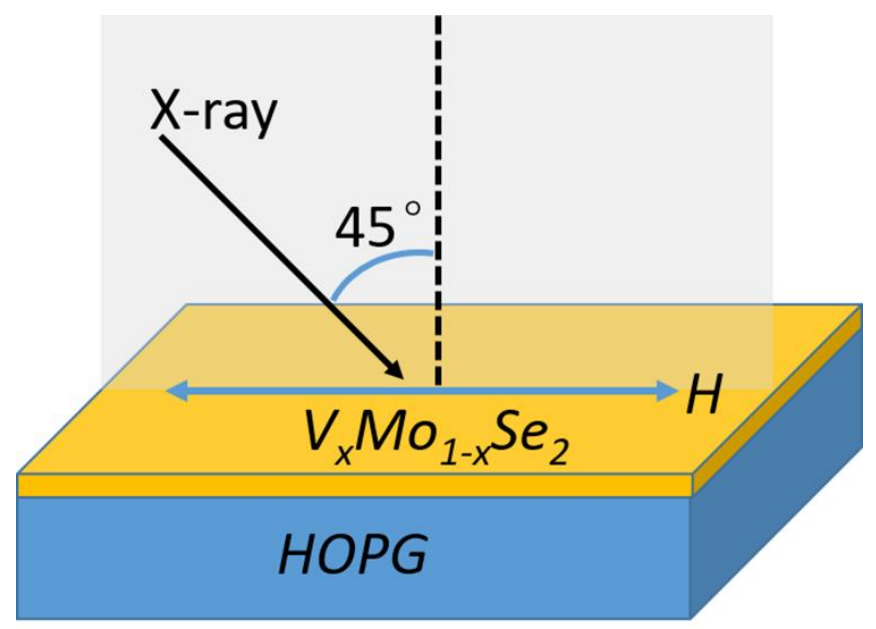

Figure S1. XAS and XMCD measurement geometry. The incident angle of the circularly polarized Xray beam with a fixed helicity of $75 \%$ was set to $45^{\circ}$ relative to the sample normal. The XAS of V $L_{2,3^{-}}$ edge were measured at $65 \mathrm{~K}$ at remanence, after the applications of opposite magnetic fields pulses parallel to the sample surface of $500 \mathrm{Oe}\left(\mathrm{H}^{+}\right)$and $-500 \mathrm{Oe}\left(\mathrm{H}^{-}\right)$at each energy step. The difference between these spectra yields the XMCD signals $\left(\mathrm{H}^{+}-\mathrm{H}^{-}\right)$. 


\section{STS of $\mathrm{V}_{0.26} \mathrm{Mo0}_{0.74} \mathrm{Se}_{2}$}

The atomic resolution STM image of the bright domains is shown in Figure S2a, in which regular atomic arrangement far from the boundaries can be faintly distinguished. A detailed STS study taken across the boundaries between bright and dark domains further confirms the band gap of $1.0 \pm 0.1 \mathrm{eV}$ of the dark domains (spots 7, 8, 9 and 10 in Figure S2c,d). The STS near the boundaries (spots 5, 6, 11 and 12 in Figure S2c,d) varies dramatically, which should be caused by the relatively inhomogeneous local distribution of $\mathrm{V}$ atoms. MTBs still can be observed in some areas, as shown in Figure S2b.
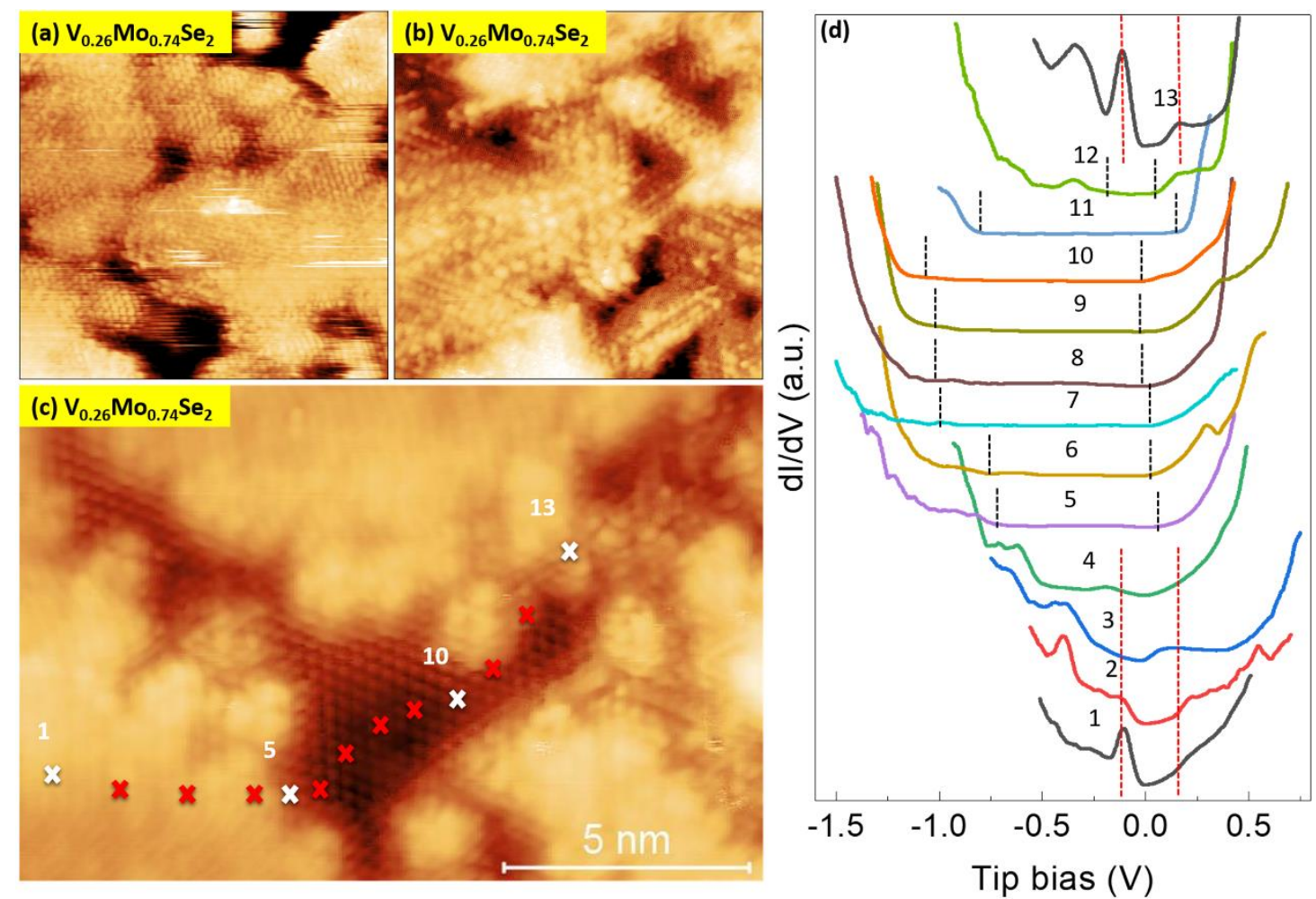

Figure S2. STM and STS of $\mathrm{V}_{0.26} \mathrm{Mo}_{0.74} \mathrm{Se}_{2}$. (a) STM image of some bright domains showing a relatively ordered lattice structure especially for those areas far from boundaries. (b) STM image of some areas showing MTBs. (d) A series of STS taken at the spots indicated by the numbered crosses in (c), showing the electronic evolution across the boundaries between bright and dark domains. Clearly, the STS of the bright domains (spots 1, 2, 3, 4 and 13) show a metallic feature, while those of the dark domains (spots $7,8,9$ and 10) show a semiconducting feature with a band gap of $1.0 \pm 0.1 \mathrm{eV}$. The band gap decreases 
dramatically when approaching the boundaries (spots 5, 6,11 and 12). Compared to that of the pure $\mathrm{MoSe}_{2}$, the band gap of the dark domains is smaller, which should be due to the slight doping by V. (Size: a, $15 \times 15 \mathrm{~nm}^{2}$; b, $12 \times 12 \mathrm{~nm}^{2}$; c, $15 \times 10 \mathrm{~nm}^{2}$. Setpoints: a, $-0.3 \mathrm{~V}, 510 \mathrm{pA}$; b, $0.4 \mathrm{~V}, 720 \mathrm{pA}$; c, $0.6 \mathrm{~V}, 350 \mathrm{pA})$ 


\section{Detailed XPS evolution as a function of $V$ concentration $x$}

The XPS of Mo $3 d$ and Se $3 d$ from $\mathrm{MoSe}_{2}$ phase almost vanish when $x=0.44$ $\left(\mathrm{V}_{0.44} \mathrm{Mo}_{0.56} \mathrm{Se}_{2}\right)$, indicating that the critical $\mathrm{V}$ concentration $x$ for phase separation is $\sim 0.44$.
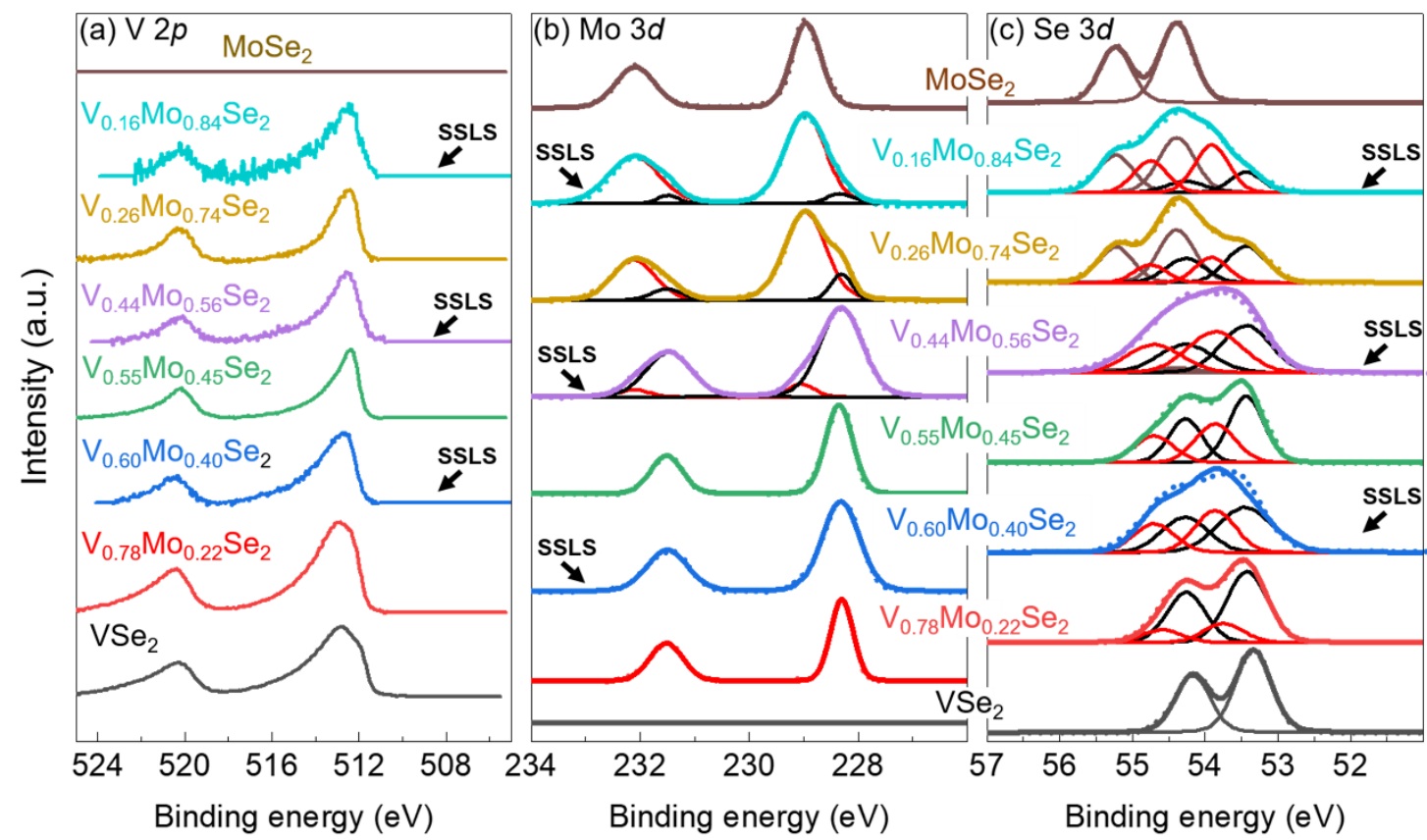

Figure S3. XPS evolution of (a) V 2p, (b) Mo $3 d$ and (c) Se $3 d$ as a function of V concentration $x$. All spectra were subtracted by a Shirley background. For (b) and (c), the experimental data are presented by shot dotted lines, envelopes of fitted peaks by thick solid lines and component peaks by thin solid lines. The $\mathrm{V} 2 p$ spectrum of pure $\mathrm{MoSe}_{2}$ and $\mathrm{Mo} 3 d$ spectrum of pure $\mathrm{VSe}_{2}$ are just pure straight lines drawn for illustration purpose. Spectra arrowed by "SSLS" were obtained at SSLS, Singapore with an $\mathrm{X}$-ray energy of $600 \mathrm{eV}$ while the other spectra were obtained at Elettra, Italy with an X-ray energy of $700 \mathrm{eV}$. Due to the lower resolution and larger noise, spectra obtained at SSLS are not as good as those obtained at Elettra. As shown in (b) and (c), the XPS of Mo $3 d$ and Se $3 d$ from $\mathrm{MoSe}_{2}$ phase almost vanish when $x=0.44\left(\mathrm{~V}_{0.44} \mathrm{Mo}_{0.56} \mathrm{Se}_{2}\right)$, indicating that the critical $\mathrm{V}$ concentration $x$ for phase separation is $\sim 0.44$. 


\section{Statistics on 457 ATAT-generated $2 \mathrm{H}-$ and 69 1T-V ${ }_{x} \mathrm{Mo1}_{-x} \mathrm{Se}_{2}$ structures}
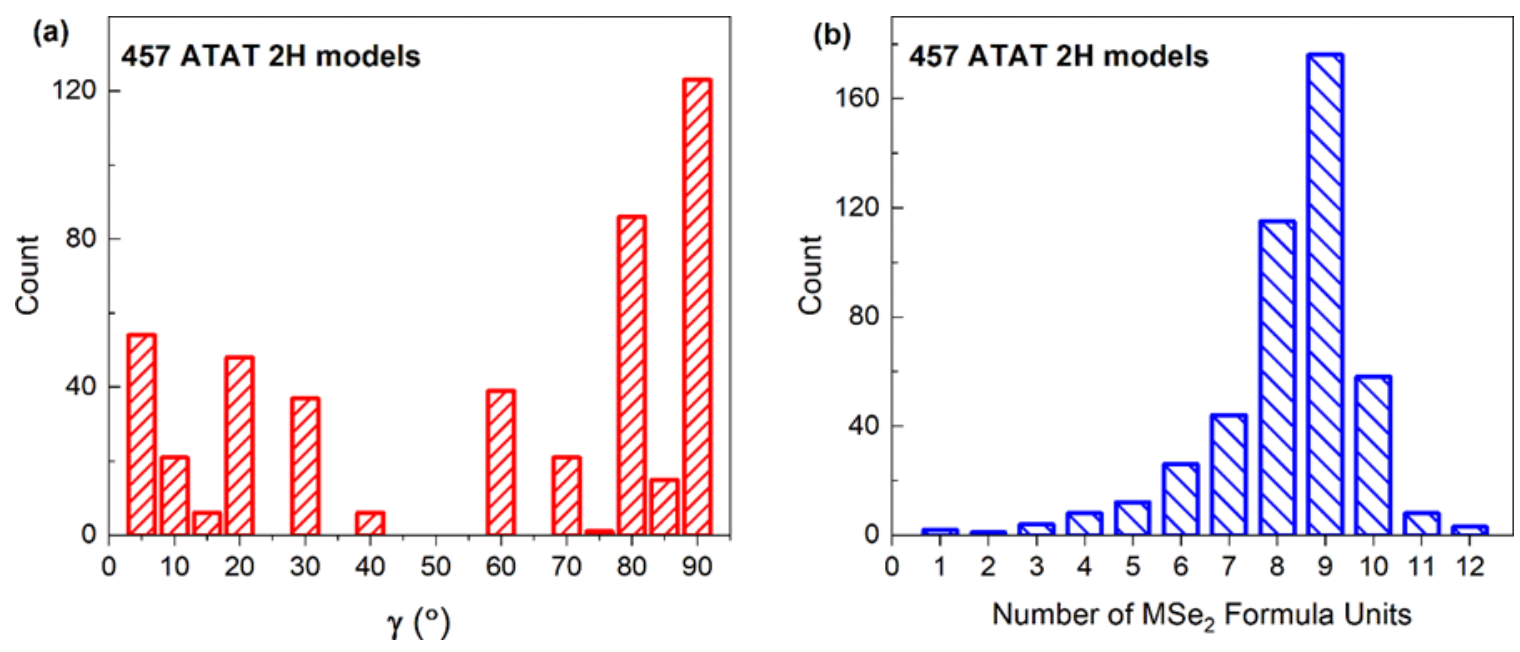

Figure S4. Distributions of (a) the angle $(\gamma)$ between the in-plane lattice vectors and (b) the number of $\mathrm{MSe}_{2}$ formula units of the 457 ATAT-generated $2 \mathrm{H}-\mathrm{V}_{x} \mathrm{Mo}_{1-x} \mathrm{Se}_{2}$ structures.
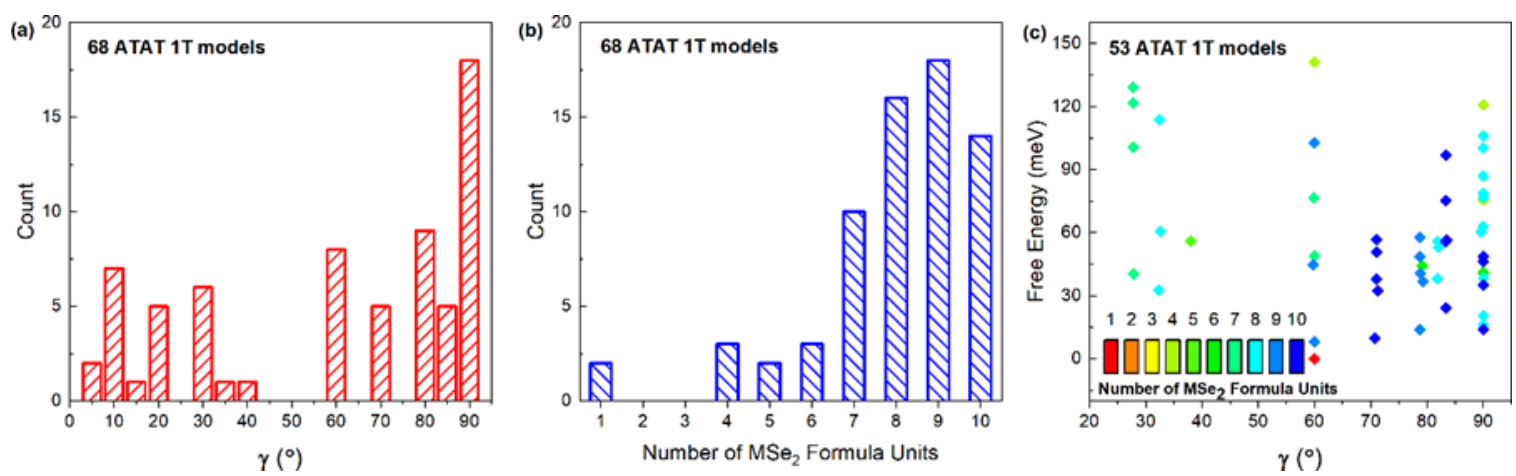

Figure S5. Distributions of (a) the angle $(\gamma)$ between the in-plane lattice vectors and (b) the number of $\mathrm{MSe}_{2}$ formula units of the 68 ATAT-generated $1 \mathrm{~T}-\mathrm{V}_{x} \mathrm{Mo}_{1-x} \mathrm{Se}_{2}$ structures. (c) The free energy per $\mathrm{MSe}_{2}$ formula unit as a function of $\gamma$. Note that 15 structures with $\gamma \leq 22^{\circ}$ have been discarded (Figure S7 and Figure S8).

In the present study, we utilized the Alloy Theoretical Automated Toolkit (ATAT) to generate structures for $\mathrm{V}_{x} \mathrm{Mo}_{1-x} \mathrm{Se}_{2}$, including 457 2H-phase and 68 1T-phase structures, in total. It is noted that ATAT tends to generate cells that are as small as possible. As such, 
a bias toward a certain cell shape may indeed occur if few structures are acquired at a given vanadium concentration $x$. To check for this issue, we analyzed the angle $(\gamma)$ between the in-plane lattice vectors of the $4572 \mathrm{H}-\mathrm{V}_{x} \mathrm{Mo}_{1-x} \mathrm{Se}_{2}$ structures studied, revealing $\gamma$ varing from $\sim 6^{\circ}$ to $90^{\circ}$, as illustrated in Figure S4a. Figure $\mathbf{6 b}$ further shows the cell shape diversity of these $2 \mathrm{H}$-phase structures at a fixed cell size. Here, these analyses can safely rule out a significant bias toward specific cell shapes among the 457 ATAT-generated $2 \mathrm{H}-$ $\mathrm{V}_{\mathrm{x}} \mathrm{Mo}_{1-\mathrm{x}} \mathrm{Se}_{2}$ structures. Compared with the $2 \mathrm{H}$-phase structures, the bias is slightly pronounced for the $681 \mathrm{~T}-\mathrm{V}_{x} \mathrm{Mo}_{1-x} \mathrm{Se}_{2}$ structures (see Figure S5).

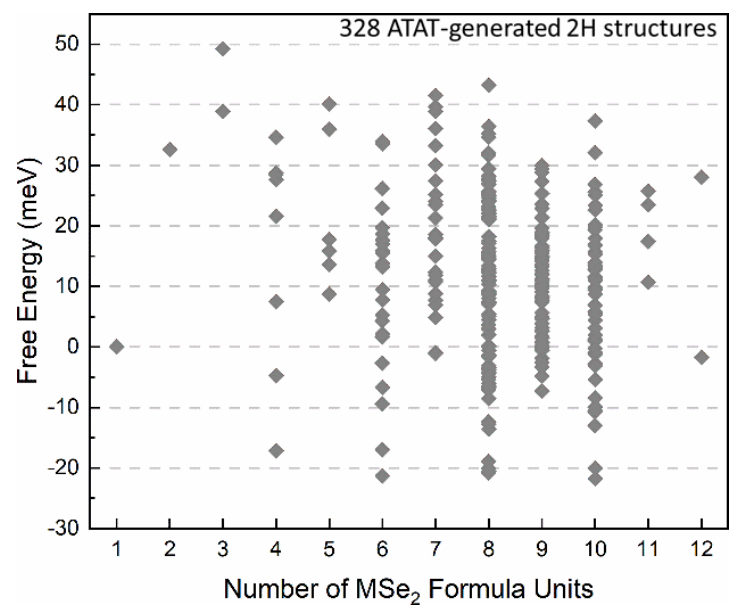

Figure S6. Free energy per formula unit of the 328 ATAT-generated $2 \mathrm{H}-\mathrm{V}_{x} \mathrm{Mo}_{1-x} \mathrm{Se}_{2}$ structures as a function of the number of $\mathrm{MSe}_{2}$ formula units. Of 457 ATAT $2 \mathrm{H} \mathrm{V}_{x} \mathrm{Mo}_{1-x} \mathrm{Se}_{2}$ structures, 129 structures with $\gamma \leq 22^{\circ}$ have been discarded (see the discussion in Figures $\mathbf{S 7}$ and S8). 


\section{Cell shape-dependent calculation accuracy}

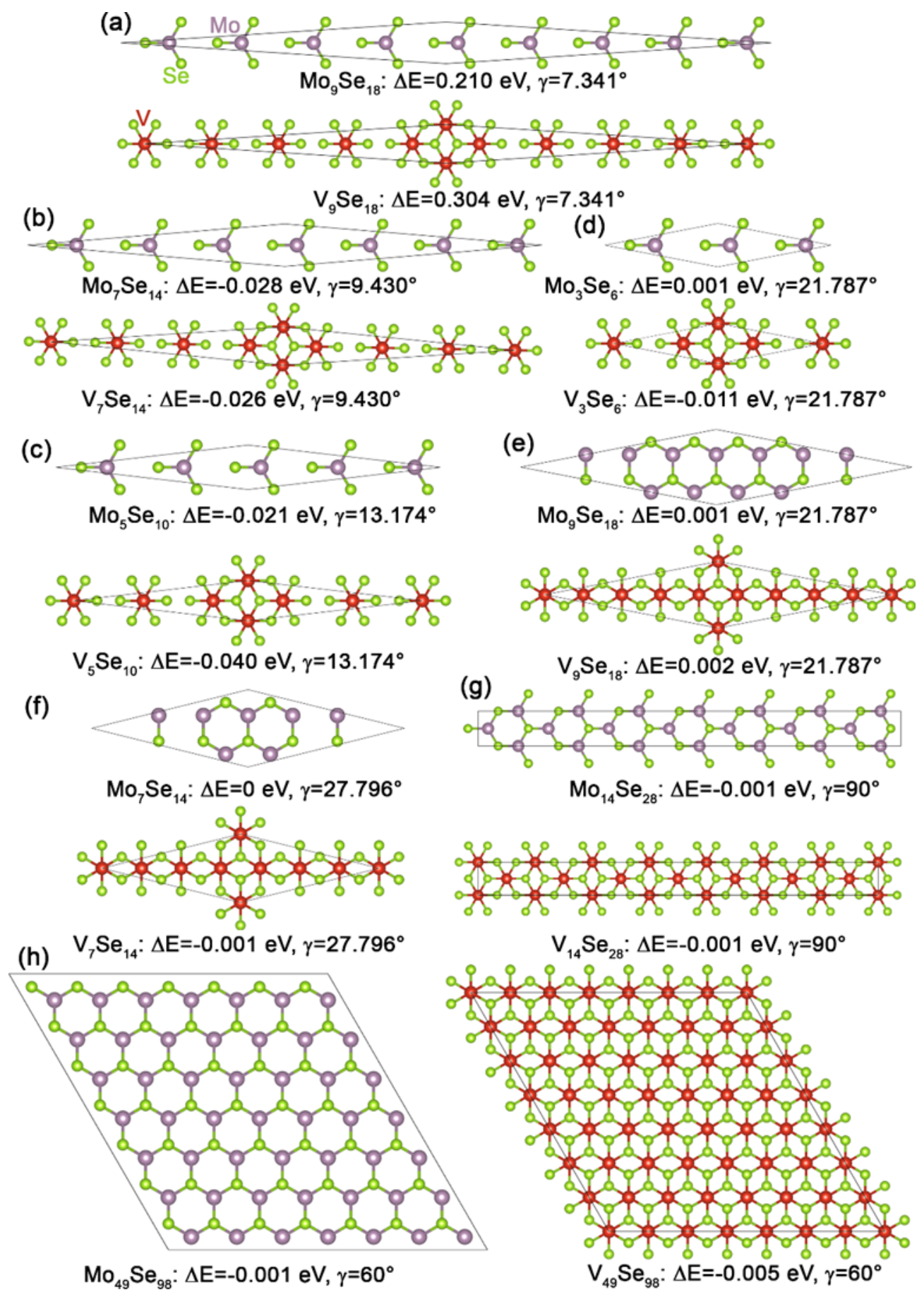

Figure S7. Comparison of the calculated energy per formula unit $(E)$ of $2 \mathrm{H}-\mathrm{MoSe}_{2}$ and $1 \mathrm{~T}-\mathrm{VSe}_{2}$ using different cells. $\Delta E$ is the difference between the energy calculated using these cells and that calculated using unit cells $\left(2 \mathrm{H}-\mathrm{MoSe}_{2}:-22.512,1 \mathrm{~T}-\mathrm{VSe}_{2}:-20.301 \mathrm{eV}\right) . \gamma$ is the angle between the in-plane lattice 
vectors. Except for $\mathrm{M}_{14} \mathrm{Se}_{28}$ and $\mathrm{M}_{48} \mathrm{Se}_{49}$, these cells appear in the $4572 \mathrm{H}-\mathrm{V}_{x} \mathrm{Mo}_{1-x} \mathrm{Se}_{2}$ and $681 \mathrm{~T}-$ $\mathrm{V}_{x} \mathrm{Mo}_{1-x} \mathrm{Se}_{2}$ structures generated by ATAT. Upon comparison, it is found that $E\left(2 \mathrm{H}-\mathrm{MoSe}_{2}\right)$ and $E(1 \mathrm{~T}-$ $\mathrm{VSe}_{2}$ ) calculated using cells with $\gamma \leq 22^{\circ}$ have significant numerical errors, hence 129 structures with $\gamma \leq 22^{\circ}$ have been discarded in the calculation of the free energy per formula unit of the ATATgenerated 2H-V $\mathrm{V}_{x} \mathrm{Mo}_{1-x} \mathrm{Se}_{2}$ structures (See Figure S5c, Figure S6 and Figure 6a,b).
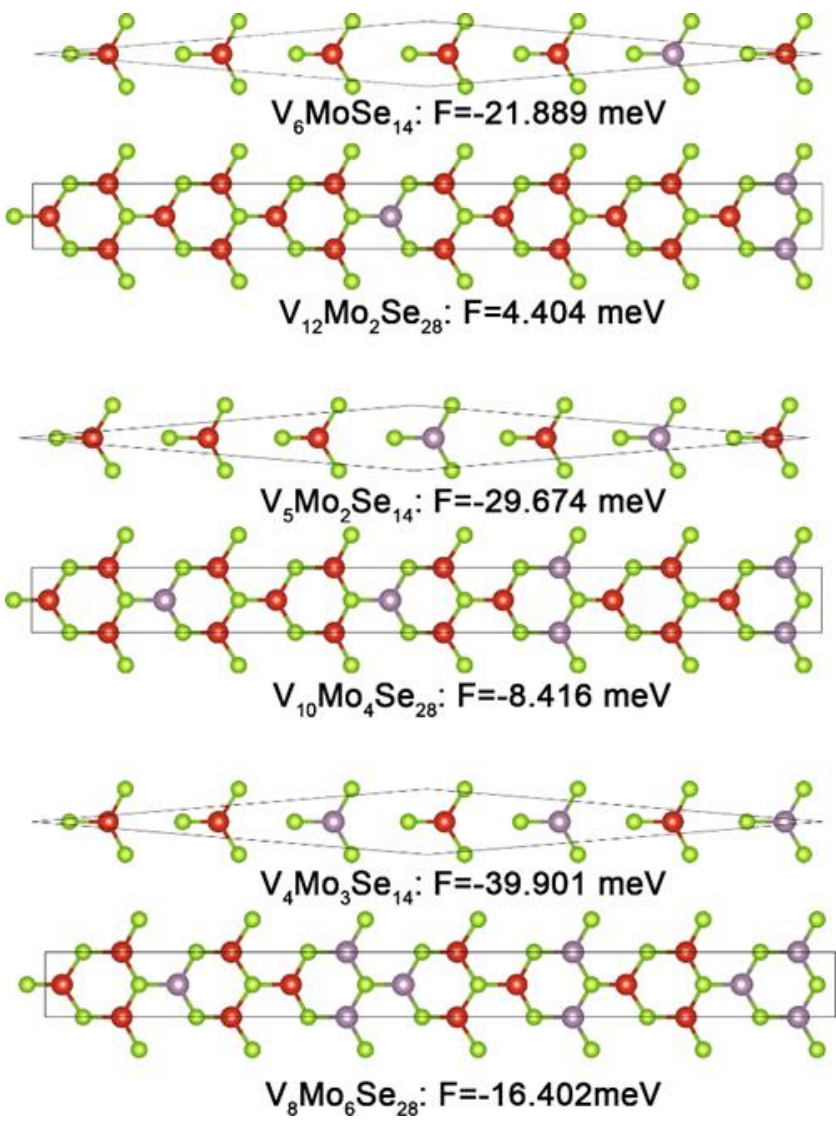

Figure S8. Comparison of the calculated free energies (F) of $\mathrm{V}_{x} \mathrm{Mo}_{1-x} \mathrm{Se}_{2}$ using a very linear cell with $\gamma \approx 9.430^{\circ}$ and the $14 \mathrm{FR}$ cell. Note that the free energies of $\mathrm{V}_{x} \mathrm{Mo}_{1-x} \mathrm{Se}_{2}$ are calculated with respect to the $2 \mathrm{H}-\mathrm{MoSe}_{2}$ and $1 \mathrm{~T}-\mathrm{VSe} \mathrm{S}_{2}$ of the same cell shapes. 


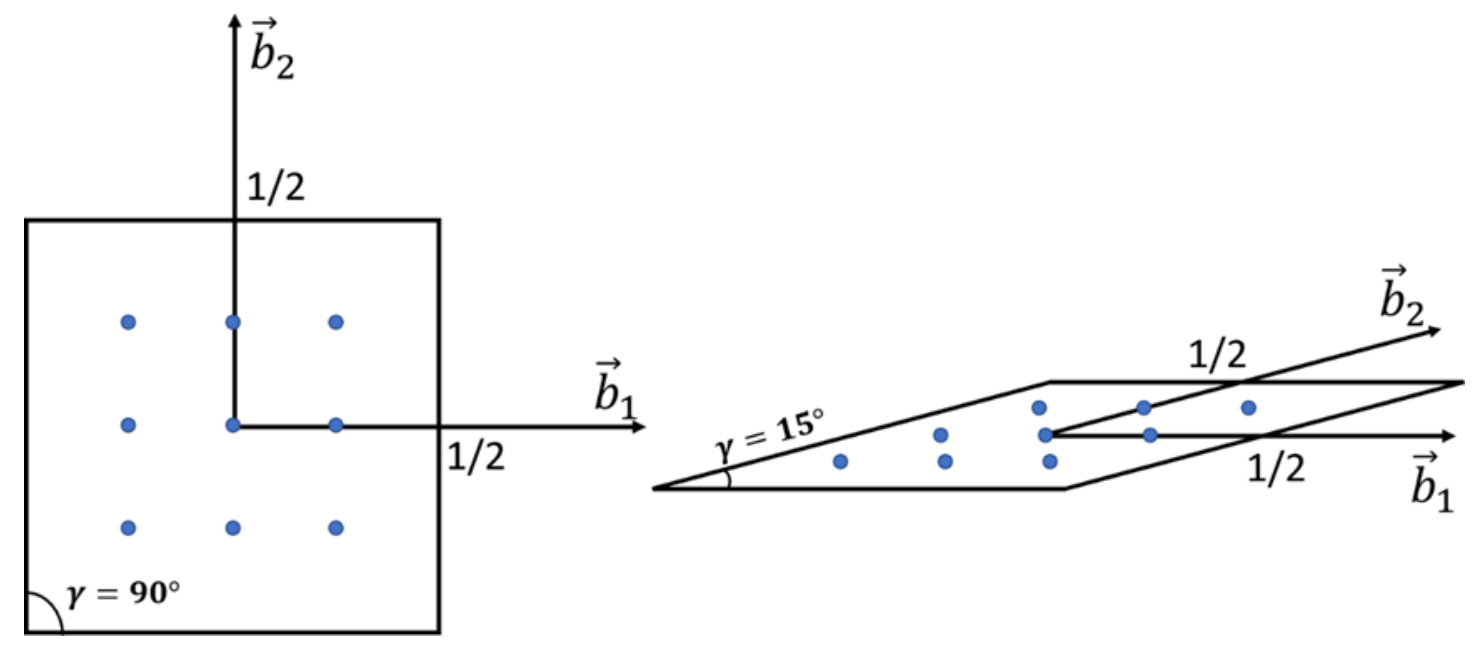

Figure S9. The $\Gamma$-centred Monkhorst-Pack schemed $k$-point sampling of the reciprocal space of two cells with the same in-plane lattice constant but with different angles $(\gamma)$ between the in-plane lattice vectors. The $k$-mesh is $3 \times 3$ and the blue dots are the sampled $k$-points. $\vec{b}_{1}$ and $\vec{b}_{2}$ are the reciprocal lattice vectors. Note that the angle between $\vec{b}_{1}$ and $\vec{b}_{2}$ is also $\gamma$. For a very linear cell with small $\gamma$, the Monkhorst-Pack scheme may not efficiently sample the reciprocal space as suggested by the unequal spacing between the $k$-points. As such, a much denser $k$-points sampling (a higher computational cost) for a very linear cell is needed to achieve the same calculation accuracy as the one on the left panel.

As shown in Figure S4 and Figure S5, the cell shape of the 457 2H- and 68 1T$\mathrm{V}_{x} \mathrm{Mo}_{1-x} \mathrm{Se}_{2}$ generated by ATAT varies in a wide range. In particular, a large amount of them have $\gamma \leq 30^{\circ}$. After careful analysis, we found that the cell shape can strongly influence the accuracy of energy calculations for very linear structures. This is apparent when looking at the energy per formula unit $(E)$ of $2 \mathrm{H}-\mathrm{MoSe}_{2}$ and $1 \mathrm{~T}-\mathrm{VSe}_{2}$ of a few cell shapes in those ATAT-generated 2H-phase and 1T-phase $\mathrm{V}_{\mathrm{x}} \mathrm{Mo}_{1-\mathrm{x}} \mathrm{Se}_{2}$ structures. For example, in Figure S7, $E\left(2 \mathrm{H}-\mathrm{MoSe}_{2}\right)$ and $E\left(1 \mathrm{~T}-\mathrm{VSe}_{2}\right)$ calculated using very linear cells with $\gamma \leq 22^{\circ}$ are seen to significantly deviate from those by unit cell calculations $\left(2 \mathrm{H}-\mathrm{MoSe}_{2}:-22.512 \mathrm{eV}, 1 \mathrm{~T}-\mathrm{VSe} \mathrm{e}_{2}\right.$ : 
$-20.301 \mathrm{eV})$. As a result, the calculated free energy of a very linear $\mathrm{V}_{x} \mathrm{Mo}_{1-x} \mathrm{Se}_{2}$ structure may not be accurate, even by referring to the $2 \mathrm{H}-\mathrm{MoSe}_{2}$ and $1 \mathrm{~T}-\mathrm{VSe}_{2}$ of the same cell shape (see Figure S8). We speculate such an issue to be related to the Monkhorst-Pack scheme being not efficient in sampling the reciprocal space (or the Brillouin zone) of very linear structures. This is illustrated in Figure S9, where the Monkhorst-Pack schemed $k$-points are much less equally spaced for very linear cells (small $\gamma$ ) than for non-linear cells, in particular along the two diagonal directions. An efficient sampling scheme would have sampled equally spaced $k$-points, otherwise. In view of such an issue of the ATAT package, we decided to discard $1292 \mathrm{H}$ - and $151 \mathrm{~T}-\mathrm{V}_{\mathrm{x}} \mathrm{Mo}_{1-\mathrm{x}} \mathrm{Se}_{2}$ structures with $\gamma \leq 22^{\circ}$ during the free energy evaluation (Figure 6a,b and Figure S5). 


\section{Statistics on the randomly generated $7 \times 7$ and $14 F R V_{x} M_{01-x} S_{2}$ structures}

Table S1. Statistics on the randomly generated structures using $7 \times 7$ and $14 \mathrm{FR}$ supercells for $2 \mathrm{H}$-phase $\mathrm{V}_{x} \mathrm{Mo}_{1-x} \mathrm{Se}_{2}$.

\begin{tabular}{cccccc}
\hline $7 \times 7$ & $\mathbf{V}$ & Number of & & \multicolumn{1}{c}{$\mathbf{V}$} & Number of \\
& Concentration & Structures & & Concentration & Structures \\
\hline $\mathrm{V}_{7} \mathrm{Mo}_{42} \mathrm{Se}_{98}$ & 0.14 & 24 & $\mathrm{~V}_{2} \mathrm{Mo}_{12} \mathrm{Se}_{28}$ & 0.14 & 7 \\
\hline $\mathrm{V}_{14} \mathrm{Mo}_{35} \mathrm{Se}_{98}$ & 0.29 & 28 & $\mathrm{~V}_{4} \mathrm{Mo}_{10} \mathrm{Se}_{28}$ & 0.29 & 73 \\
\hline $\mathrm{V}_{21} \mathrm{Mo}_{28} \mathrm{Se}_{98}$ & 0.43 & 27 & $\mathrm{~V}_{6} \mathrm{Mo}_{8} \mathrm{Se}_{28}$ & 0.43 & 217 \\
\hline $\mathrm{V}_{22} \mathrm{Mo}_{27} \mathrm{Se}_{98}$ & 0.45 & 24 & $\mathrm{~V}_{8} \mathrm{Mo}_{6} \mathrm{Se}_{28}$ & 0.57 & 217 \\
\hline $\mathrm{V}_{28} \mathrm{Mo}_{21} \mathrm{Se}_{98}$ & 0.57 & 31 & $\mathrm{~V}_{10} \mathrm{Mo}_{4} \mathrm{Se}_{28}$ & 0.71 & 73 \\
\hline $\mathrm{V}_{35} \mathrm{Mo}_{14} \mathrm{Se}_{98}$ & 0.71 & 25 & $\mathrm{~V}_{12} \mathrm{Mo}_{2} \mathrm{Se}_{28}$ & 0.86 & 7 \\
\hline $\mathrm{V}_{42} \mathrm{Mo}_{7} \mathrm{Se}_{98}$ & 0.86 & 25 & & & \\
\hline $\mathrm{Total}^{2}$ & 184 & & & 594 \\
\hline
\end{tabular}

Table S2. The number of low free-energy configurations $\left(n_{\text {conf }}\right)$ and average number of $\mathrm{V}$ and Mo strips $\left(<n_{\mathrm{V} \mid \text { Mo strips }}>\right.$ ) in these low free-energy configurations among the $14 \mathrm{FR} \mathrm{V}_{x} \mathrm{Mo}_{1-x} \mathrm{Se}_{2}$ structures. The energy window is set to 5 and $10 \mathrm{meV}$ with respect to the lowest-energy structure at each $\mathrm{V}$ concentration $x . n_{\mathrm{V}}$ and $n_{\mathrm{Mo}}$ are the number of $\mathrm{V}$ and Mo atoms in the 14FR structures, respectively.

\begin{tabular}{|c|c|c|c|c|c|}
\hline$n_{\mathrm{V}}$ & V concentration & & $5 \mathrm{meV}$ & & $10 \mathrm{meV}$ \\
\hline & & $n_{\text {conf }}$ & $<n_{\mathrm{V} \mid \text { Mo strips }}>$ & $n_{\text {conf }}$ & $<n_{\mathrm{V} \mid \text { Mo strips }}>$ \\
\hline $2 / 14$ & 0.14 & 1 & 2 & 1 & 2 \\
\hline $4 / 14$ & 0.29 & 6 & 3.667 & 15 & 3.867 \\
\hline $6 / 14$ & 0.43 & 19 & 4.632 & 32 & 4.750 \\
\hline $8 / 14$ & 0.57 & 27 & 5.259 & 41 & 5.269 \\
\hline $10 / 14$ & 0.71 & 27 & 5.259 & 36 & 5.278 \\
\hline $12 / 14$ & 0.86 & 6 & 3.667 & 7 & 3.714 \\
\hline
\end{tabular}




\section{XMCD of $\mathrm{V}_{x} \mathrm{Mo1}_{-x} \mathrm{Se}_{2}$ alloys}

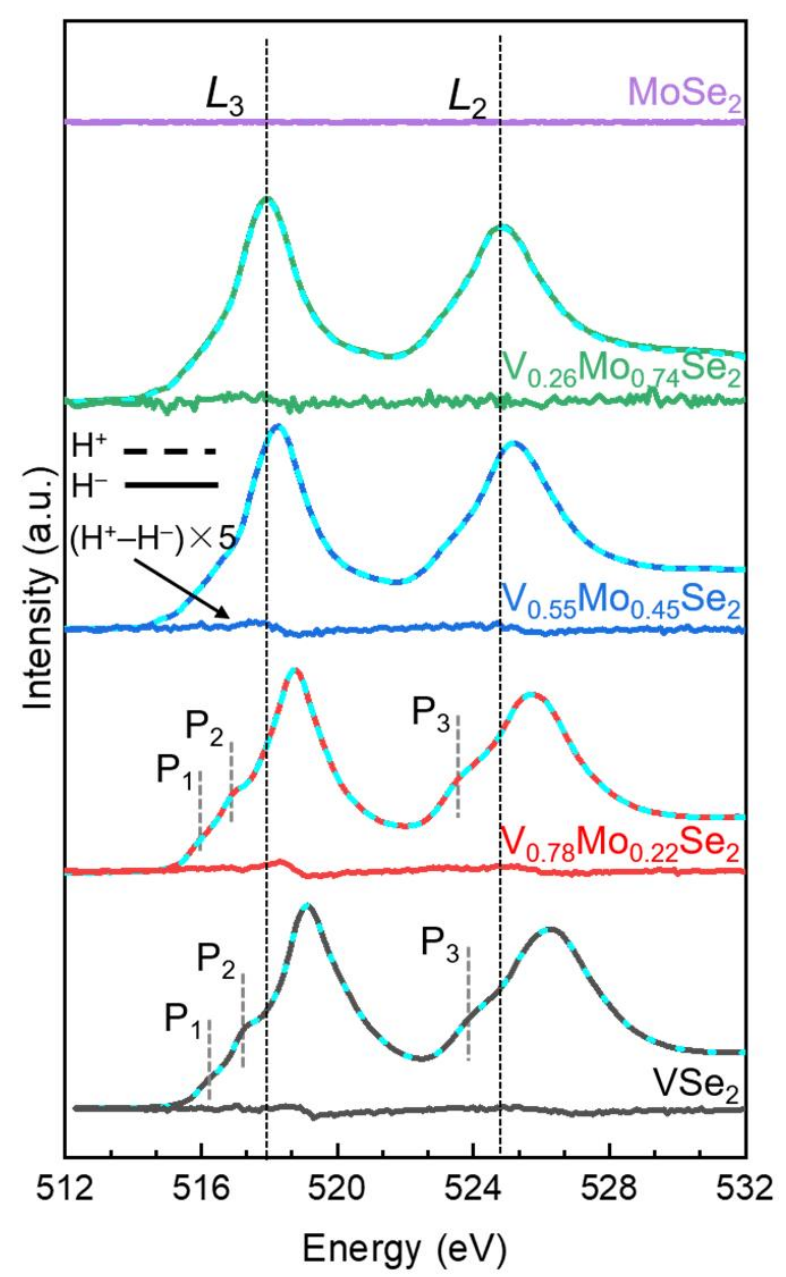

Figure S10. XAS and XMCD. The XAS of V $L_{2,3}$-edge obtained at remanence after in-plane magnetic field pulses of $500 \mathrm{Oe}\left(\mathrm{H}^{+}\right)$and $-500 \mathrm{Oe}\left(\mathrm{H}^{-}\right)$at $\mathrm{T}=65 \mathrm{~K}$ were plotted in short dashed and solid lines, respectively. The corresponding XMCD signals $\left(\mathrm{H}^{+}-\mathrm{H}^{-}\right)$were magnified and displayed below correspondingly, showing the absence of ferromagnetism. All spectra were normalized to constant height. Fine structures in XAS are indicated by P1, P2 and P3.

While no theoretical calculations have been directed to explore the magnetic properties of $\mathrm{V}_{x} \mathrm{Mo}_{1-x} \mathrm{Se}_{2}$ alloys, a previous DFT study has indicated local magnetic moments in the constituting $\mathrm{V}$ atoms of a similar alloy, $\mathrm{V}_{x} \mathrm{~W}_{1-x} \mathrm{Se}_{2} .{ }^{1} \mathrm{XMCD}$ signals $\left(\mathrm{H}^{+}-\right.$ $\mathrm{H}^{-}$) are obtained and shown in Figure S10. Within the experimental error, the XMCD of alloys with $x=0.26,0.55$ and 0.78 are below the instrumental sensitivity limit (less than $0.1 \%$ ), hence cannot confirm the presence of ferromagnetism. The measured XMCD value 
for monolayer $\mathrm{VSe}_{2}$ (less than $0.1 \%$ ) also shows no ferromagnetic signal in our experimental conditions, which is consistent with previous XMCD and angle-resolved photoemission spectroscopy (ARPES) measurements, ${ }^{2,3}$ but conflicts with calculations, ${ }^{4-9}$ superconducting quantum interference device (SQUID) and longitudinal magneto-optic Kerr effect (L-MOKE) magnetometer measurements. ${ }^{10,11}$ Thus, both the existence and origin of ferromagnetism in monolayer $\mathrm{VSe}_{2}$ need further investigation.

\section{References:}

(1) Manchanda, P.; Skomski, R. 2D Transition-Metal Diselenides: Phase Segregation, Electronic Structure, and Magnetism. J. Phys. Condens. Matter 2016, 28, 064002.

(2) Feng, J.; Biswas, D.; Rajan, A.; Watson, M. D.; Mazzola, F.; Clark, O. J.; Underwood, K.; Marković, I.; McLaren, M.; Hunter, A.; Burn, D. M.; Duffy, L. B.; Barua, S.; Balakrishnan, G.; Bertan, F.; Fèvre, P. L; Kim, T. K.; van der Laan, G.; Hesjedal, T.; Wahl, P.; et al. Electronic Structure and Enhanced Charge-Density Wave Order of Monolayer VSe2. Nano Lett. 2018, 18, 4493-4499.

(3) Wong, P. K. J.; Zhang, W.; Bussolotti, F.; Yin, X.; Herng, T. S.; Zhang, L.; Huang, Y. L.; Vinai, G.; Krishnamurthi, S.; Bukhvalov, D. W.; Zheng, Y. J.; Chua, R.; N'Diaye, A. T.; Morton, S. A.; Yang, C.-Y.; Ou Yang, K.-H.; Torelli, P.; Chen, W.; Goh, K. E. J.; Ding, J.; et al. Evidence of Spin Frustration in a Vanadium Diselenide Monolayer Magnet. Adv. Mater. 2019, 31, 1901185.

(4) Ma, Y.; Dai, Y.; Guo, M.; Niu, C.; Zhu, Y.; Huang, B. Evidence of the Existence of Magnetism in Pristine $\mathrm{VX}_{2}$ Monolayers $(\mathrm{X}=\mathrm{S}, \mathrm{Se})$ and Their Strain-Induced Tunable Magnetic Properties. ACS Nano 2012, 6, 1695-1701.

(5) Fuh, H.-R.; Chang, K.-W.; Hung, S.-H.; Jeng, H.-T. Two-Dimensional Magnetic 
Semiconductors Based on Transition-Metal Dichalcogenides $\mathrm{VX}_{2}(\mathrm{X}=\mathrm{S}, \mathrm{Se}, \mathrm{Te})$ and Similar Layered Compounds $\mathrm{VI}_{2}$ and $\mathrm{Co}(\mathrm{OH})_{2}$. IEEE Magn. Lett. 2016, 8, 3101405 .

(6) Esters, M.; Hennig, R. G.; Johnson, D. C. Dynamic Instabilities in Strongly Correlated VSe 2 Monolayers and Bilayers. Phys. Rev. B 2017, 96, 235147.

(7) Popov, Z. I.; Mikhaleva, N. S.; Visotin, M. A.; Kuzubov, A. A.; Entani, S.; Naramoto, H.; Sakai, S.; Sorokin, P. B.; Avramov, P. V. The Electronic Structure and Spin States of 2D Graphene $/ \mathrm{VX}_{2}(\mathrm{X}=\mathrm{S}, \mathrm{Se})$ Heterostructures. Phys. Chem. Chem. Phys. 2016, $18,33047-33052$.

(8) Fuh, H.-R.; Yan, B.; Wu, S.-C.; Felser, C.; Chang, C.-R. Metal-Insulator Transition and the Anomalous Hall Effect in the Layered Magnetic Materials $\mathrm{VS}_{2}$ and $\mathrm{VSe}_{2}$. New J. Phys. 2016, 18, 113038.

(9) Xu, K.; Chen, P.; Li, X.; Wu, C.; Guo, Y.; Zhao, J.; Wu, X.; Xie, Y. Ultrathin Nanosheets of Vanadium Diselenide: A Metallic Two-Dimensional Material with Ferromagnetic Charge-Density-Wave Behavior. Angew. Chemie - Int. Ed. 2013, 52, 10477-10481.

(10) Bonilla, M.; Kolekar, S.; Ma, Y.; Diaz, H. C.; Kalappattil, V.; Das, R.; Eggers, T.; Gutierrez, H. R.; Phan, M.-H.; Batzill, M. Strong Room-Temperature Ferromagnetism in $\mathrm{VSe}_{2}$ Monolayers on van der Waals Substrates. Nat. Nanotechnol. 2018, 13, 289-293.

(11) Duvjir, G.; Choi, B. K.; Jang, I.; Ulstrup, S.; Kang, S.; Thi Ly, T.; Kim, S.; Choi, Y. H.; Jozwiak, C.; Bostwick, A.; Rotenberg, E.; Park, J.-G.; Sankar, R.; Kim, K.-S.; 
Kim, J.; Chang, Y. J. Emergence of a Metal-Insulator Transition and HighTemperature Charge-Density Waves in $\mathrm{VSe}_{2}$ at the Monolayer Limit. Nano Lett. 2018, $18,5432-5438$. 